\title{
Optimal interpretation of Tc99m PYP in 2020: Avoiding the million-dollar mistake
}

\author{
Jaime Gerber, $M D{ }^{a}$ and Edward J. Miller, $M D, P h D^{a}$ \\ a Section of Cardiovascular Medicine, Yale University School of Medicine, New Haven, CT
}

Received Sep 28, 2020; accepted Sep 28, 2020

doi: $10.1007 / \mathrm{s} 12350-020-02503-8$

\section{See related article, doi: https://doi.org/1 0.1007/s12350-020-02328-5.}

The diagnosis of transthyretin cardiac amyloidosis (ATTR-CA) is now literally a million-dollar implication. With the approval of tafamidis (Vyndaqel/ Vyndamax) in 2019, ATTR-CA has become a treatable chronic disease state with potentially life prolonging therapy for those properly diagnosed and with access to this revolutionary treatment. ${ }^{1}$ This treatment comes at a significant expense to patients and society as the direct lifetime cost of treatment with tafamidis for ATTR-CA was recently calculated to be 1.13 million dollars per patient, ${ }^{2,3}$ and there are unanswered questions about its cost-effectiveness. ${ }^{2}$ As nuclear scintigraphy with bone-avid tracers $\left({ }^{99 \mathrm{~m}} \mathrm{Tc}-\mathrm{py}-\right.$ rophosphate $\left({ }^{99 \mathrm{~m}} \mathrm{Tc}-\mathrm{PYP}\right)$ in the United State) is now central to the diagnosis of ATTR-CA eliminating the need for endomyocardial biopsy in most cases, careful consideration of ATTR-CA imaging techniques and interpretation is of foremost clinical importance to our patients and also has major economic impact for the world healthcare budget. It is crucial that ${ }^{99 \mathrm{~m}} \mathrm{Tc}-\mathrm{PYP}$ imaging be accurately interpreted: Mis-diagnosing ATTR-CA through ${ }^{99 m}$ Tc-PYP misinterpretation is now a 'million-dollar mistake.'

The rapid increase in use of ${ }^{99 \mathrm{~m}}$ Tc-PYP imaging for the diagnosis ATTR-CA over the past decade developed around the concept that a refreshingly straightforward imaging scheme utilizing planar imaging provides high diagnostic yield. ${ }^{4,5}$ The visual interpretive approach to

Reprint requests: Edward J. Miller, MD, PhD, Section of Cardiovascular Medicine, Yale University School of Medicine, New Haven, CT ; edward.miller@yale.edu

J Nucl Cardiol 2021;28:503-6.

$1071-3581 / \$ 34.00$

Copyright (C) 2021 American Society of Nuclear Cardiology. planar ${ }^{99 \mathrm{~m}}$ Tc-PYP imaging uses a 0-3 grading system of the appearance of cardiac uptake on the anterior and lateral projections adopted from ${ }^{99 \mathrm{~m}} \mathrm{Tc}-3,3$-diphosphono-1,2-propanodicarboxylic acid ( $\left.{ }^{99 \mathrm{~m}} \mathrm{Tc}-\mathrm{DPD}\right)$ imaging. ${ }^{6}$ This was further supplemented in 2013 by the work by Bokhari et al. who developed the semiquantitative heart/contralateral chest ratio to differentiate ATTR-CA from light chain cardiac amyloidosis (ALCA) in appropriately selected patients. ${ }^{4}$ Additively, the seminal publication by Gilmore et al. described that the combination of visual grade 2 or 3 myocardial radiotracer uptake on planar bone scintigraphy and the absence of a monoclonal protein in serum or urine had a specificity and positive predictive value for ATTR-CA of $100 \% .^{5}$ The patient population in this study consisted of highly selected patients evaluated at tertiary amyloid referral centers with expertise in the performance and interpretation of bone scintigraphy for many years. However, as the contemporary utilization of ${ }^{99 \mathrm{~m}}$ Tc-PYP imaging expands to the community through efforts of nuclear cardiology societies and pharmaceutical companies seeking to expand the reach of their targeted therapies, the patients undergoing ${ }^{99 \mathrm{~m}}$ Tc-PYP imaging now reflect a less selected population with a likely much lower pre-test probability of ATTR-CA. While there are little data on the rapidly evolving practice of ${ }^{99 m}$ Tc-PYP imaging, recent practice surveys indicate that at least $30 \%$ of responding hospitals perform Tc-PYP imaging to screen for cardiac amyloidosis, but often times without light chain measurement to exclude AL-CA and only $21 \%$ of institutions performed SPECT imaging in addition to planar. ${ }^{7}$ These data and anecdotal reports from referral centers suggest the rapid expansion of ${ }^{99 m}$ Tc-PYP imaging to non-expert centers may be leading to reduced interpretive specificity of ${ }^{99 \mathrm{~m}} \mathrm{Tc}-\mathrm{PYP}$ imaging for ATTR-CA both due to testing of patients that have low probability of ATTR-CA/high probability of alternative diagnoses (such as AL-CA) and imprecise acquisition/interpretation strategies. It seems reasonable to assume that this increased utilization of ${ }^{99 m}$ Tc-PYP 
imaging will continue to expand in the future into even lower-risk populations that include screening of asymptomatic 'at risk' patients, making it even more important for nuclear cardiologist to have the most specific approaches to acquiring and interpreting bone scintigraphy for ATTR-CA.

Limitations to the diagnostic specificity of planaronly ${ }^{99 \mathrm{~m}} \mathrm{Tc}$-PYP imaging when performed following a 1-hour incubation and interpreted only by visual assessment and/or semiquantitative $\mathrm{H} / \mathrm{CL}$ ratios are increasingly recognized. These limitations include increased apparent cardiac uptake on planar images as a result of increased blood pool activity, acute or subacute myocardial infarction, low cardiac output, pleural effusions, obesity, bone uptake such as from rib fractures, presence of mitral or aortic calcification, and hydroxychloroquine toxicity among other etiologies. ${ }^{8}$ Technologist and physician inexperience with $\mathrm{H}: \mathrm{CL}$ region of interest (ROI) placement are another significant contributors to inaccurate $\mathrm{H}: \mathrm{CL}$ ratio. The effect of this reduced specificity is the misdiagnosis of some patients using the 'non-invasive' pathway, with the resultant possibility that these patients will be prescribed extraordinarily expensive anti-TTR therapies in error.

Therefore, to improve specificity, adaptations to the ${ }^{99 \mathrm{~m}}$ Tc-PYP imaging protocol have been proposed including extending the duration of the incubation to 2-3 hours and adding SPECT (and preferably SPECT/CT) acquisitions. Lengthening the incubation period to 3 hours allows blood pool clearance but with a drawback of increased bone uptake and a subsequent lower 'positive' H/CL ratio (approximately 1.3 for 3 hours vs 1.5 for 1 hour). The benefits of SPECT imaging include ensuring apparent cardiac uptake seen on planar imaging is truly in the myocardium, validating that the cardiac uptake is diffuse, and potentially allowing for future use of quantitative tools such as standardized uptake values (SUVs) that could enhance prognosis. Furthermore, SPECT/CT can improve interpretive certainty by the review of the fused SPECT and CT images to improve confidence in the anatomic localization of ${ }^{99 \mathrm{~m}} \mathrm{Tc}$-PYP uptake in the thorax. Therefore, SPECT imaging (with or without CT) has emerged as the 'gold standard' for determining ${ }^{99 \mathrm{~m}}$ Tc-PYP positivity, realizing that no study has reported the accuracy of ${ }^{99 \mathrm{~m}}$ Tc-PYP SPECT vs biopsy confirmation. The downsides of SPECT acquisitions include increased imaging time for the patient, reduced lab efficiency (although minimal), and technical difficulty with SPECT reconstructions when myocardial uptake is absent.

In the current report in the journal by Asif et al., ${ }^{9}$ they retrospectively analyzed the diagnostic accuracy of planar imaging to the additional accuracy afforded by SPECT imaging in a single-center cohort of 133 patients. Focusing on specificity, they showed that 2 out of 98 patients were misclassified as positive if planar visual score alone was used and 1 was misclassified as positive if $\mathrm{H}: \mathrm{CL}$ was used. Highlighting problems with $\mathrm{H}: \mathrm{CL}$ quantification, they also found $\mathrm{H}: \mathrm{CL}$ to be insensitive as it was $<1.5$ (negative) in 15 out of 35 $(43 \%)$ patients that were SPECT positive.

These data build on two other recent reports that compared 1- vs 3-hour imaging and planar vs SPECT acquisitions and their effects on diagnostic accuracy. ${ }^{10,11}$ The data from all three reports are summarized in Table 1. While the protocols, patient populations, and equipment were not identical in these articles, some important and consistent findings emerge. First and most importantly, none of the two publications comparing 1and 3-hour imaging ${ }^{10,11}$ found a difference in any patients' $(\mathrm{n}=242){ }^{99 \mathrm{~m}}$ Tc-PYP interpretation when comparing 1- and 3-hour SPECT. This 100\% concordance between 1- and 3-hour ${ }^{99 \mathrm{~m}} \mathrm{Tc}$-PYP SPECT imaging is important if we are to consider SPECT to be the 'gold standard' for cardiac ${ }^{99 \mathrm{~m}} \mathrm{Tc}-\mathrm{PYP}$ uptake. On the contrary, there are known to be significant differences between cardiac uptake and $\mathrm{H}: \mathrm{CL}$ ratios on 1-and 3-hour 'planar'-only imaging studies for reasons discussed above and demonstrated in these studies in Table 1. Second, all the reports consistently find that the use of $\mathrm{H}: \mathrm{CL}$ ratio as a sole diagnostic criterion has a lower PPV compared to planar imaging. Third, all of the reports show that SPECT imaging has a small, but measurable, improvement in positive predictive value compared to visual planar analysis alone.

These data create important considerations for nuclear cardiology imaging practice. They make clear that 1-hour planar-only imaging is not reliable and should not be used. Using Table 1 as a guide, 1-hour planar-only imaging would lead to approximately $2 \%$ to $7 \%$ of studies being read as false positive. SPECT imaging should be used in all cases, with SPECT/CT preferred if it is available. The issue of incubation time (1- vs 3-hours) is potentially more nuanced. Given the data from Sperry et al. and Masri et al. showing no difference in the SPECT interpretations between images acquired at the different time points, it is reasonable to suggest that a 1-hour imaging approach that incorporates experienced technologists, readers, and (preferably) SPECT/CT at referral centers familiar with the pre-test probability of ATTR-CA in their patients and interpretation of ${ }^{99 \mathrm{~m}}$ Tc-PYP is very reasonable. However, it is clear that many centers will not meet these criteria. With the practical concern that in many ambulatory settings scheduling a three-hour delayed study will remove a perfusion imaging slot from the schedule and require that the patient return to the lab after the three-hour waiting period has passed, a reasonable alternative is for 
Table 1. Comparison of three recent studies comparing acquisition and interpretation parameters of ${ }^{99 \mathrm{~m}}$ Tc-PYP imaging for ATTR-CA

\begin{tabular}{|c|c|c|c|}
\hline & Asif et al. ${ }^{?}$ & Sperry et al. ${ }^{11}$ & Masri et al. ${ }^{10}$ \\
\hline$N$ & 133 & 109 & 233 \\
\hline Diagnosed ATTR (+) (\%) & 26 & 30 & 26 \\
\hline 1-Hour imaging & Yes & Yes & Yes \\
\hline 3-Hour imaging & No & Yes & Yes \\
\hline $\operatorname{SPECT}(\mathrm{Y} / \mathrm{N})$ & Yes & Yes (SPECT/CT) & Yes \\
\hline \multicolumn{4}{|l|}{1 hour } \\
\hline \multicolumn{4}{|l|}{ Discordant results } \\
\hline SPECT $(-) /$ visual $\geq 2$ & 2 & 2 & 7 \\
\hline SPECT $(-) / \mathrm{H}: C L \geq 1.5$ & 1 & 2 & 16 \\
\hline \multicolumn{4}{|l|}{ Visual $\geq 2^{*}$} \\
\hline Sens. (\%) & 97 & $81 \#$ & 98 \\
\hline Spec. & 98 & 96 & 96 \\
\hline PPV & 94 & 93 & 89 \\
\hline \multicolumn{4}{|l|}{$\mathrm{H}: \mathrm{CL} \geq 1.5^{*}$} \\
\hline Sens. (\%) & 57 & 88 & 97 \\
\hline Spec. & 95 & 97 & 91 \\
\hline PPV & 80 & 93 & 78 \\
\hline \multicolumn{4}{|l|}{3 hour } \\
\hline \multicolumn{4}{|l|}{ Discordant results } \\
\hline SPECT $(-) /$ visual $\geq 2$ & $\mathrm{~N} / \mathrm{A}$ & 1 & 1 \\
\hline $\operatorname{SPECT}(-) / \mathrm{H}: \mathrm{CL} \geq 1.5$ & $\mathrm{~N} / \mathrm{A}$ & 11 & 10 \\
\hline \multicolumn{4}{|l|}{ Visual $\geq 2^{*}$} \\
\hline Sens. (\%) & & $91 \#$ & 93 \\
\hline Spec. & $\mathrm{N} / \mathrm{A}$ & 98 & 99 \\
\hline PPV & & 97 & 98 \\
\hline \multicolumn{4}{|l|}{$\mathrm{H}: \mathrm{CL} \geq 1.5^{*}$} \\
\hline Sens. (\%) & & 94 & 95 \\
\hline Spec. & $\mathrm{N} / \mathrm{A}$ & 88 & 94 \\
\hline PPV & & 74 & 85 \\
\hline
\end{tabular}

* Using SPECT as gold-standard reference

\#Sperry et al. combined visual and H:CL interpretation in results

an $\mathrm{MD}$ reader to review the one-hour imaging, including planar and SPECT. If there is any question regarding the degree of myocardial uptake and/or blood pool, then 3hour delayed planar and SPECT imaging is required. Furthermore, training for technologists in how to acquire and process reconstructions of "empty chests" and for readers to identify blood pool and bone is needed. A potential "is delayed imaging needed?" imaging approach utilized at our laboratories is depicted in Figure 1. This approach assumes an experienced reader is able to interpret and decide upon the necessity of a longer incubation in real time.

In summary, the diagnostic approach to ATTR-CA using ${ }^{99 \mathrm{~m}} \mathrm{Tc}-\mathrm{PYP}$ imaging (and other bone-avid tracers) is one of the modern-day success stories in nuclear cardiology. In less than a decade, this testing strategy has revolutionized the evaluation and care of patients with heart failure with preserved ejection fraction, enabling an etiologic diagnosis and indication for treatment where previously none existed. However, as with any area of rapidly evolving change in practice, we need to be prepared to step back and refine our approaches based on emerging data. In the case of ${ }^{99 \mathrm{~m}}$ Tc-PYP imaging for ATTR-CA based on the data by Asif et al. and others, it is clear that SPECT imaging and careful attention to longer incubation times to improve specificity are now mandatory. These approaches will ensure we appropriately diagnose ATTR-0CA in those 


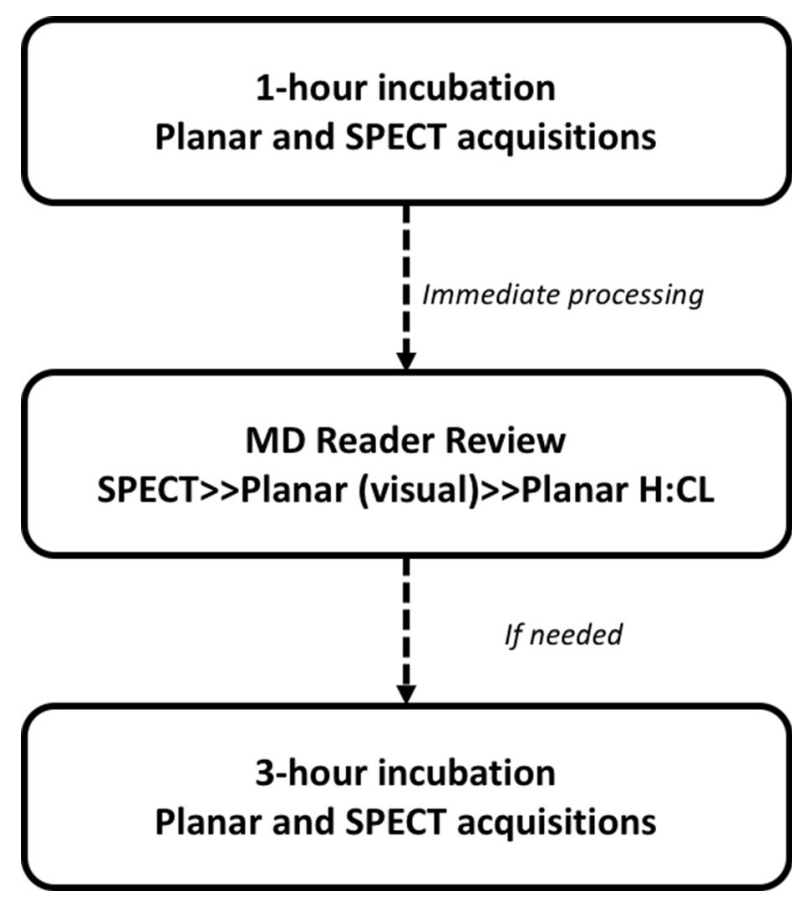

Figure 1. Proposed incorporation of 1- and 3-hour imaging protocols for ${ }^{99 \mathrm{~m}} \mathrm{Tc}$-PYP imaging for ATTR-CA. If a 1-hour interpretation is performed, interpreting physicians should focus on the presence of diffuse myocardial uptake and absence of excessive blood pool on SPECT image to confirm diagnosis. Planar visual uptake can be used to provide a grade of cardiac PYP uptake intensity. Planar H:CL ratios require technologist and reader expertise to ensure accuracy. Threehour imaging should be pursued if the interpretation of the 1hour images is not certain and/or there is limited laboratory expertise with ${ }^{99 \mathrm{~m}}$ Tc-PYP imaging.

that have the disease and avoid giving million-dollar treatments to those that do not.

\section{References}

1. Maurer MS, Schwartz JH, Gundapaneni B, et al. Tafamidis treatment for patients with transthyretin amyloid cardiomyopathy. N Engl J Med. 2018;379(11):1007-16.

2. Kazi DS, Bellows BK, Baron SJ, et al. Cost-effectiveness of tafamidis therapy for transthyretin amyloid cardiomyopathy. Circulation. 2020;73:2872.

3. Masri A, Masri A, Chen $\mathrm{H}$, et al. Initial experience prescribing commercial tafamidis, the most expensive cardiac medication in history. JAMA Cardiol. 2020;5:1066-7.

4. Bokhari S, Castaño A, Pozniakoff T, et al. (99m)Tc-pyrophosphate scintigraphy for differentiating light-chain cardiac amyloidosis from the transthyretin-related familial and senile cardiac amyloidoses. Circ Cardiovasc Imaging. 2013;6:195-201.

5. Gillmore JD, Maurer MS, Falk RH, et al. Nonbiopsy diagnosis of cardiac transthyretin amyloidosis. Circulation. 2016;133:2404-12.

6. Perugini E, Guidalotti PL, Salvi F, et al. Noninvasive etiologic diagnosis of cardiac amyloidosis using 99mTc-3,3-diphosphono1,2-propanodicarboxylic acid scintigraphy. J Am Coll Cardiol. 2005;46:1076-84.

7. Harb SC, Haq M, Flood K, et al. National patterns in imaging utilization for diagnosis of cardiac amyloidosis: A focus on Tc99m-pyrophosphate scintigraphy. J Nucl Cardiol. 2017;24:1094-7.

8. Hanna M, Ruberg FL, Maurer MS, et al. Cardiac Scintigraphy With Technetium-99m-labeled bone-seeking tracers for suspected amyloidosis: JACC review topic of the week. J Am Coll Cardiol. 2020;75:2851-62.

9. Asif T, Gomez J, Singh V, et al. Comparison of planar with tomographic pyrophosphate scintigraphy for transthyretin cardiac amyloidosis: Perils and pitfalls. J Nucl Cardiol. 2020. https://doi. org/10.1007/s12350-020-02328-5.

10. Masri A, Bukhari S, Ahmad S, et al. Efficient 1-hour technetium$99 \mathrm{~m}$ pyrophosphate imaging protocol for the diagnosis of transthyretin cardiac amyloidosis. Circ Cardiovasc Imaging. 2020. h ttps://doi.org/10.1161/CIRCIMAGING.119.010249.

11. Sperry BW, Burgett E, Bybee KA, et al. Technetium pyrophosphate nuclear scintigraphy for cardiac amyloidosis: Imaging at 1 vs 3 hours and planar vs SPECT/CT. J Nucl Cardiol. 2020. h ttps://doi.org/10.1007/s12350-020-02139-8.

Publisher's Note Springer Nature remains neutral with regard to jurisdictional claims in published maps and institutional affiliations. 